\title{
Clinical practice of caesarean section revisited: present and future
}

Noel WM Shek *, MRCOG, FHKAM (Obstetrics and Gynaecology)

Department of Obstetrics and Gynaecology, Queen Mary Hospital, The University of Hong Kong, Pokfulam, Hong Kong

Hong Kong Med J 2017;23:324-5

* shekwmn@ha.org.hk

DOI: $10.12809 / \mathrm{hkmj} 175068$

Historically, the introduction of caesarean section (CS) was associated with an improvement in maternal and perinatal health outcomes. In 1985, the World Health Organization (WHO) recommended that CS should not account for more than $10 \%$ to $15 \%$ of all births. ${ }^{1}$ The WHO has recently revised their position and stated that "every effort should be made to provide a CS to women in need, rather than striving to achieve a specific rate." ${ }^{2}$ The effect of CS rates on other outcomes-such as maternal and perinatal morbidity, paediatric outcomes, and psychological or social well-being-are still unclear. ${ }^{2}$ Of note, CS carries its own risks for maternal and infant morbidity and for subsequent pregnancies. At some point, these risks will outweigh the potential benefits associated with lowering the threshold at which the procedure becomes indicated.

In recent decades, there has been a rising tide in CS worldwide with a wide variation in CS rate among various countries from approximately $16 \%$ to more than $60 \% .^{3}$ The reasons for the increase in CS rates are multiple and complex, but have been attributed to the increasing prevalence of older mothers, rising rates of maternal obesity and medical co-morbidities, and changing medical practice including a relative increased safety of CS itself. ${ }^{4-7}$ In addition, there is substantial evidence that this increase is more prevalent among women with privately funded deliveries. ${ }^{8,9}$ Nevertheless, the dramatic rise in CS rate has not been shown to be accompanied by any substantial decrease in maternal or perinatal morbidity or mortality..$^{10}$ Malpractice litigation pressure has been suggested as one of the attributes for the rise because associations have been demonstrated between CS rates and malpractice premiums. ${ }^{11}$

In Hong Kong, the annual CS rate rose steadily from $16.6 \%$ to $27.4 \%$ from 1987 to 1999 , with the rate in private institutions of $27.4 \%$ higher than the public sector. ${ }^{9}$ Published in this issue of the Hong Kong Medical Journal, a retrospective review of CS rates from 1995 to 2014 at a local public hospital by Chung et $\mathrm{al}^{12}$ shows that the overall rate increased modestly from $15.4 \%$ to $24.6 \%$. Nonetheless, it is well known by women in Hong Kong that government-funded units under the Hospital Authority do not perform elective CS for non-clinical indications. Those with a strong preference for elective CS might seek private maternity care. The territory-wide audit conducted by the Hong Kong College of Obstetricians and
Gynaecologists has documented an increase in overall CS rates in Hong Kong from 27.1\% in 1999 to $30.4 \%$ in 2004 and $42.1 \%$ in $2009 .^{13}$ The latest annual obstetric report of the Hospital Authority in 2015 showed CS rates in the eight public hospitals in Hong Kong varying from $21.7 \%$ to $30.4 \%$. $^{14}$

The WHO recently adopted the Robson's classification system as a global standard for assessing, monitoring, and comparing CS rates. ${ }^{2}$ Robson's system classifies women into 10 groups based on five obstetric characteristics that are routinely documented: parity, onset of labour, gestational age, fetal presentation, and number of fetuses. The actual indication for CS is not needed for categorisation. The categories in Robson's system are mutually exclusive, totally inclusive, and can be applied prospectively. ${ }^{15}$ It allows comparison of clinically meaningful maternity population subgroups and their associated CS rates across institutions, country development groups, and time. The Robson's classification has been used to analyse trends and determinants of CS rates in high- and low-income countries, such as the data analysis of 21 countries included in the WHO survey. ${ }^{16}$ The retrospective review by Chung et $\mathrm{al}^{12}$ used the Robson's classification system to categorise a 20 -year database up to 2014. It showed dramatic and statistically significant increases $(\mathrm{P}<0.001)$ in CS rate in those with previous CS (rising from $29 \%$ to $61 \%$ ), breech presentation at delivery (primiparous from $72 \%$ to $97 \%$ and multiparous from $69 \%$ to $96 \%$ ), and multiple pregnancies (from $35 \%$ to $86 \%$ ). The authors suggested that the rise in the previous CS group was secondary to a more liberal policy to allow patients to choose CS after abandonment of pelvimetry to predict successful trial of labour. The increased CS rate in breech presentation group may be due to publication of the Term Breech Trial in 2000, whereas the increase in the multiple pregnancy group was attributed to the liberal policy that accommodated patient expectations. Nonetheless, a significant fall from $14 \%$ to $11 \%$ was noted in the group of primiparous patients with term spontaneous labour. Such progressive drop in CS rate was a result of the adoption of evidence-based active management of labour protocols, and regular audits in CS rates and indications within the unit.

A local cross-sectional survey of 660 Chinese pregnant women in a government-funded obstetric 
unit in Hong Kong found that previous CS and conception by in-vitro fertilisation were significant determinants of a preference for elective CS. ${ }^{17}$ In another local retrospective cohort study of twin pregnancies, conception by assisted reproduction was also a statistically significant factor that affected maternal preference for elective CS. ${ }^{18}$ Non-cephalic presentation of the second twin was another statistically significant factor in the study, indicating women's concern for their babies when considering mode of delivery. The survey also showed that women who preferred elective CS were concerned about safety of the baby, and feared a vaginal birth and the pain associated with the delivery.

Two randomised controlled trials aimed to determine whether interventions were useful to reduce the number of women seeking CS. One focused on using an individualised prenatal educational programme in women with previous $\mathrm{CS}^{19}$ and the other used cognitive treatment in women who were fearful of a vaginal birth. ${ }^{20}$ Both showed no significant difference between intervention and control groups with respect to the women's request for elective CS. These results may imply that once fear is established, treatment is not of significant clinical benefit.

To reduce the overall $\mathrm{CS}$ rate, reducing the proportion of first deliveries by CS appears pertinent. Public and prenatal education may play an important role in shaping expectations. Obstetric management protocols, skills, and clinical audits can be targeted at reducing first birth by CS, eg external cephalic version in term-breech pregnancies, safe vaginal twin delivery techniques, standardised fetal heart rate tracing interpretation and management, and increasing women's access to non-medical interventions during labour such as labour and delivery support. More drastic attempts to curb primary CS rates in primiparous women can be considered, such as redefining labour dystocia, postponing the cut-off for active labour at $6-\mathrm{cm}$ dilatation, allowing adequate time for the second stage of labour, or encouraging operative vaginal delivery. ${ }^{10}$ Last but not least, obstetricians should fully discuss the risks and benefits of a vaginal birth versus CS, especially when $\mathrm{CS}$ is requested without a clinical indication. In such cases it is important to explore, discuss, and record the specific reasons for the request, and to include a discussion with other members of the obstetric team (including obstetrician, midwife, and anaesthetist) if necessary to explore the reasons for the request and ensure the woman has accurate information. ${ }^{21}$ The skill needed to make a balanced clinical decision for an individual woman may perhaps be greater than that required to undertake the procedure.

\section{References}

1. Appropriate technology for birth. Lancet 1985;2:436-7.

2. World Health Organization. WHO statement on caesarean section rates. April 2015. Available from: http://apps.who.
int/iris/bitstream/10665/161442/1/WHO_RHR 15.02 eng.pdf?ua=1. Accessed Jun 2017.

3. Visser GH. Women are designed to deliver vaginally and not by caesarean section: an obstetrician's view. Neonatalogy 2015;107:8-13.

4. O'Dwyer V, Farah N, Fattah C, O'Connor N, Kennelly MM, Turner MJ. The risk of caesarean section in obese women analysed by parity. Eur J Obstet Gynecol Reprod Biol 2011;158:28-32.

5. Brick A, Layte R. Recent trends in the caesarean section rate in Ireland 1999-2006. ESRI Working Paper No. 309. August 2009. Available from: https://www.esri.ie/pubs/WP309.pdf. Accessed Jun 2017.

6. Brick A, Layte R. Exploring trends in the rate of caesarean section in Ireland 1999-2007. Econ Soc Rev (Irel) 2011;42:383-406.

7. Bayrampour $\mathrm{H}$, Heaman M. Advanced maternal age and the risk of cesarean birth: a systematic review. Birth 2010;37:219-26.

8. Lipkind HS, Duzyj C, Rosenberg TJ, Funai EF, Chavkin W, Ciasson MA. Disparities in caesarean delivery rates and associated adverse neonatal outcomes in New York City hospitals. Obstet Gynecol 2009;113:1239-47.

9. Leung GM, Lam TH, Thach TQ, Wan S, Ho LM. Rates of cesarean births in Hong Kong: 1987-1999. Birth 2001;28:166-72.

10. American College of Obstetricians and Gynecologists (College); Society for Maternal-Fetal Medicine; Caughey AG, Cahill AG, Guise JM, Rouse DJ. Safe prevention of the primary caesarean delivery. Am J Obstet Gynecol 2014;210:179-93.

11. Yang YT, Mello MM, Subramanian SV, Studdert DM. Relationship between malpractice litigation pressure and rates of cesarean section and vaginal birth after caesarean section. Med Care 2009;47:234-42.

12. Chung WH, Kong CW, To WW. Secular trends in caesarean section rates over 20 years in a regional obstetric unit in Hong Kong. Hong Kong Med J 2017;23:340-8.

13. Territory-wide audit in obstetrics and gynaecology. Hong Kong: The Hong Kong College of Obstetricians and Gynaecologists; 2014.

14. Hospital Authority Obstetric Annual Report 2015. Hong Kong: Hospital Authority; 2016.

15. Torloni MR, Betran AP, Souza JP, et al. Classifications for cesarean section: a systematic review. PLoS One 2011;6:e14566.

16. Vogel JP, Betrán AP, Vindevoghel N, et al. Use of the Robson classification to assess caesarean section trends in 21 countries: a secondary analysis of two WHO multicountry surveys. Lancet Glob Health 2015;3:e260-70.

17. Pang SM, Leung DT, Leung TY, Lai CY, Lau TK, Chung TK. Determinants of preference for elective caesarean section in Hong Kong Chinese pregnant women. Hong Kong Med J 2007;13:100-5.

18. Liu AL, Yung WK, Yeung HN, et al. Factors influencing the mode of delivery and associated pregnancy outcomes for twins: a retrospective cohort study in a public hospital. Hong Kong Med J 2012;18:99-107.

19. Fraser W, Maunsell E, Hodnett E, Moutquin JM. Randomized controlled trial of a prenatal vaginal birth after cesarean section education and support program. Childbirth Alternatives Post-Cesarean Study Group. Am J Obstet Gynecol 1997;176:419-25.

20. Saisto T, Salmela-Aro K, Nurmi JE, Könönen T, Halmesmäki E. A randomized controlled trial of intervention in fear of childbirth. Obstet Gynecol 2001;98(5 Pt 1):820-6.

21. National Institute for Health and Care Excellence. NICE Clinical guideline: Caesarean section (CG132). 2012. Available from: https://www.nice.org.uk/guidance/cg132. Accessed Jun 2017. 